\title{
Der Aufklärer
}

URBAN DAUB Bei einer Amputation wird oft zu spät bedacht, dass sich die Belastung auf die erhaltene Extremität gravierend verändert. Das zieht häufig Gelenkbeschwerden nach sich, die sich durch gelenkschonende Verhaltensregeln reduzieren ließen. Urban Daub und seine Kollegen fanden heraus, dass es wichtig ist, die Patienten darüber aufzuklären.

Herr Daub, was schätzen Sie an der Arbeit im FraunhoferInstitut?

Ich arbeite mit anderen Berufsgruppen wie Ingenieuren und Sportwissenschaftlern zusammen. Das macht mir viel Spaß. Jeder hat seinen eigenen Blick, und gemeinsam entdecken wir Problemstellungen und entwickeln Lösungen, auf die der Einzelne nicht gekommen wäre.

Sie reisen gerne. Welches Land hat Sie am meisten begeistert? Letztes Jahr haben wir unseren Traum verwirklicht und sind nach Hawaii geflogen. Das war wirklich unbeschreiblich. Ich hoffe, das irgendwann einmal wiederholen zu können.

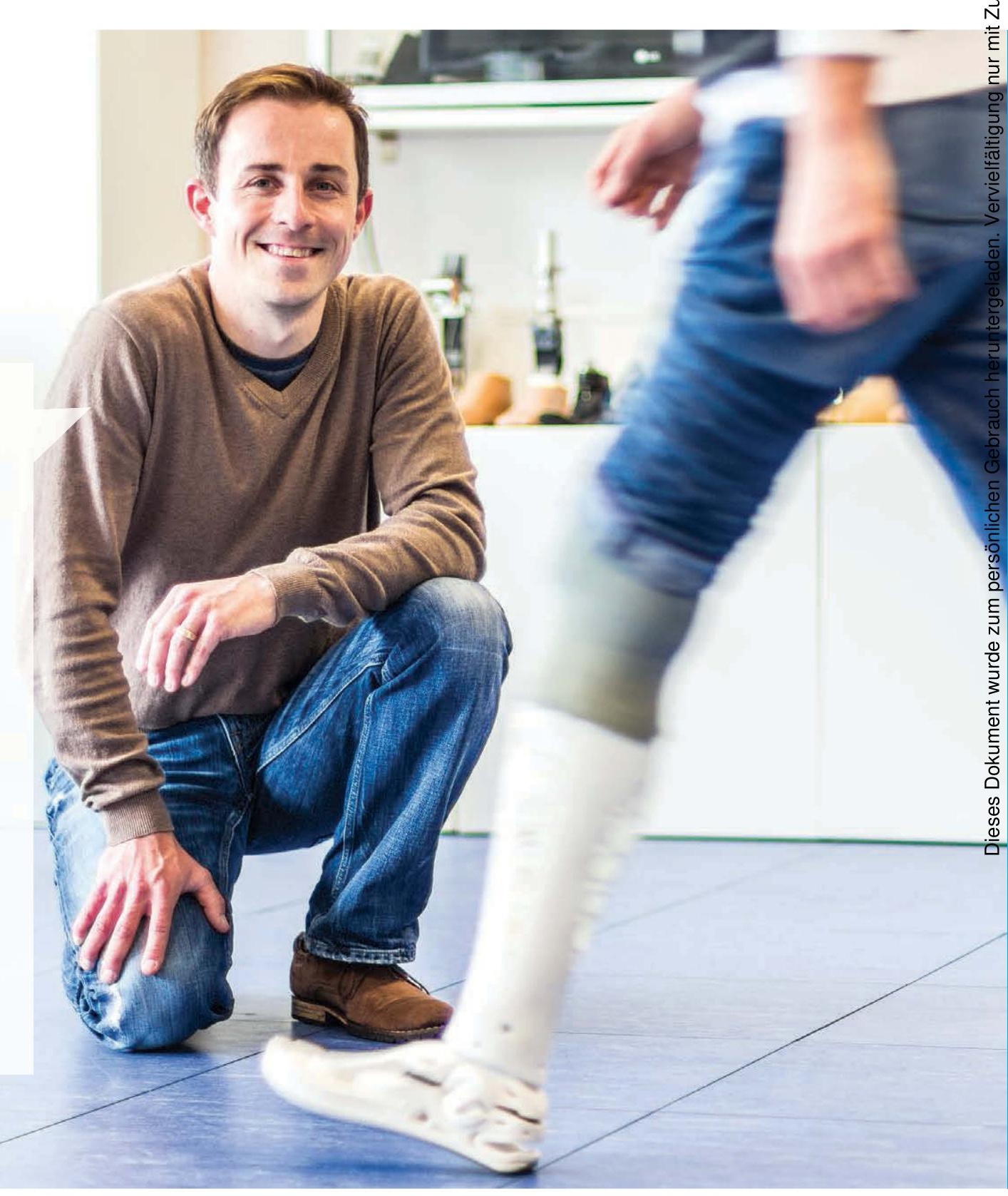




\section{Verhaltensempfehlungen für Menschen mit Amputation}

\section{Die Erhebung}

Verliert ein Patient durch eine Amputation eine Extremität, sind die restlichen Gelenke einer besonderen Belastung ausgesetzt. Durch ein verändertes Bewegungsverhalten erhöht sich der Einfluss von Scherkräften. Beim Kniegelenk etwa konnten Untersuchungen nachweisen, dass auf der nicht betroffenen Seite eines Patienten mit Beinamputation signifikant höhere Belastungen auftreten als bei vergleichbaren gesunden Probanden [1, 2]. Zudem zeigten Studien, dass sich durch diese konstante Überbelastung später vermehrt Arthrosen im Kniegelenk entwickeln [1-6]. Auch Rückenschmerzen sind in diesem Zusammenhang häufig zu finden [7-9]. Verlieren die Patienten durch die Beschwerden zusätzlich an Mobilität, verschlechtert sich ihre Selbstständigkeit oft noch weiter. Einfache präventive Regeln für ein gelenkschonendes Bewegungs- und Alltagsverhalten könnten alldem entgegenwirken.

Aus der Zusammenarbeit mit Prothesenherstellern, Selbsthilfegruppen für Menschen mit Amputation und Probanden im Prüflabor des Fraunhofer-Instituts machten Urban Daub und seine Kollegen die Erfahrung, dass Menschen mit einer Amputation solche Regeln jedoch oft nicht kennen und Therapeuten und Betreuer sie nicht darauf aufmerksam machen. Diese Erfahrung wollten sie in Zahlen belegen und sich zudem einen ersten Eindruck darüber verschaffen, welche Beschwerden bei Menschen mit Amputation auftreten. Für diese Erhebung verteilten die Forscher an zwei aufeinanderfolgenden Jahren (2012 und 2013) auf der Jahresversammlung des Bundesverbands für Menschen mit Arm- oder Beinamputation (BMAB) einen kurzen Fragebogen an die Teilnehmer. Insgesamt konnten sie 32 Fragebögen auswerten. Die befragten Patienten waren durchschnittlich 58 Jahre alt. 13 von ihnen hatten eine Unterschenkel-, 14 eine Oberschenkelamputation, drei Teilnehmer eine Knieexartikulation und eine Person eine Oberarmamputation. Ein Patient machte nur die unspezifische Angabe „Beinamputation“. Alle Eingriffe lagen im Durchschnitt 19 Jahre zurück.

\section{Zentrale Erkenntnisse}

Das Stuttgarter Team fand heraus, dass ...

$>$ ein Großteil der Befragten (etwa 80 Prozent) Gelenkbeschwerden wie Knie-, Fuß- und Rückenschmerzen hat.

$>$ nur einer der Patienten direkt nach der Amputation Verhaltensregeln erhalten hatte.

$>$ der Großteil der Befragten (84 Prozent) auch bei auftretenden Beschwerden von Therapeuten und Ärzten keine Informationen über Verhaltensregeln bekam.

> 80 Prozent der Befragten Möglichkeiten gefunden haben, ihre Beschwerden zu lindern. Dazu zählen unter anderem Physiotherapie, Bewegung, Einreibungen und Ruhepausen.

\section{Fazit}

Zusammenfassend können sie sagen, dass ...

$>$ alle Menschen nach Amputation Informationen zu Folgeerkrankungen und präventiven Verhaltensregeln erhalten sollten. Dazu wäre es zum Beispiel möglich, einen schriftlichen Handlungsleitfaden zu erstellen und den Patienten mitzugeben.

$>$ Therapeuten unter anderem folgende einfache Verhaltensregeln kennen und vermitteln sollten: Die Haut im Stumpfbereich sollte regelmäßig kontrolliert werden, Menschen mit Beinamputation sollten einbeiniges „Herumspringen“ vermeiden. Patienten mit Armamputation sollten schwere Lasten, vor allem über Gürtelniveau, vermeiden, um den erhaltenen Arm nicht zu überlasten.

$>$ viele Menschen mit Amputation bereits praktische Tipps zur Gelenkentlastung und Erleichterung bei Schmerzen im Alltag kennen. Diese Erfahrungswerte könnten zusammen mit statistischen und biomechanischen Werten die Basis eines solchen Handlungsleitfadens bilden. Eva Trompetter

$\Rightarrow$ Die Literatur steht im Artikelarchiv unter www.thieme-connect.de/products/ physiopraxis > „Ausgabe 6/15“".

$\rightarrow$ Zur Entwicklung des Leitfadens können Sie und Ihre Patienten das Stuttgarter Team in einem kurzen Onlinefragebogen unter www.amp-leitfaden.de unterstützen. Vielen Dank! 